\title{
Sensitive Electrochemical Detection of Dopamine With a Nitrogen-doped Graphene Modified Glassy Carbon Electrode
}

\author{
Wencheng Wang, ${ }^{1}$ Shixing Gong, ${ }^{2}$ Wei Sun, ${ }^{1, *}$ Guangjiu Li, ${ }^{2}$ Yaping Lu, ${ }^{2}$ Jianhua Yu, ${ }^{2}$ Lifeng Dong $2,3, *$
}

\author{
Key Laboratory of Tropical Medicinal Plant Chemistry of Ministry of Education, College of Chemistry and Chemical Engineering, Hainan Normal University, \\ Haikou 571158, P. R. China \\ 2 College of Materials Science and Engineering, Qingdao University of Science and Technology, Qingdao 266042, P. R. China \\ 3 Department of Physics, Hamline University, St. Paul, MN 55104, USA \\ * Corresponding authors' e-mail address: swyy26@hotmail.com
}

RECEIVED: June 12, 2015 * REVISED: September 12, 2016 * ACCEPTED: October 3, 2016

Abstract: In this paper nitrogen-doped graphene (NG) nanosheets were used as the modifier on the surface of glassy carbon electrode (GCE). The modified electrode (NG/GCE) was further applied to the sensitive detection of dopamine (DA) by voltammetric method. Due to the unique properties of NG such as large surface area and excellent electrocatalytic activity, electrochemical response of DA was greatly enhanced on NG/GCE with a pair of well-defined redox peaks appeared on cyclic voltammogram. Electrochemical behaviors of DA on NG/GCE were carefully investigated with the electrochemical parameters calculated. Under the selected conditions the oxidation peak currents of DA had a good linear relationship with its concentration in the range from $8.0 \times 10^{-7} \mathrm{~mol} \mathrm{~L}^{-1}$ to $8.0 \times 10^{-4} \mathrm{~mol} \mathrm{~L}^{-1}$ with a detection limit of $2.55 \times 10^{-7} \mathrm{~mol} \mathrm{~L}^{-1}(3 \sigma)$. The proposed method was further applied to the DA injection samples determination with satisfactory results.

Keywords: nitrogen-doped graphene, glassy carbon electrode, dopamine, electrochemistry.

\section{INTRODUCTION}

A $S$ a two-dimensional carbon nanomaterial with an atomically thick honeycomb lattice, graphene (GR) has aroused great interests due to its unique properties such as big surface area, high electrochemical conductivity and good biocompatibility. ${ }^{[1,2]}$ Also GR can be used as the matrix for the synthesis of different kinds of composite materials. GR and GR-based composites have been synthesized and used in various fields such as biosensor, lithium ion batteries, fuel cells and supercapacitors. ${ }^{[3,4]}$ Due to the specific electrochemical characteristics of GR, the presence of GR and its related materials on the electrode surface can provide a high conductive interface with electrocatalytic activity, which can accelerate the electron transfer rate. ${ }^{[5,6]}$ The applications of GR and its related composite in the electrochemistry had been reviewed recently. ${ }^{[7,8]}$

Recently nitrogen-doped graphene (NG) has been used in the field of electrochemistry and molecular sensing. ${ }^{[9,10]}$
As an important strategy to tailor the structure and properties of GR, chemical doping GR can be realized by different synthetic methods. ${ }^{[11]}$ Because nitrogen atom has comparable atomatic size and contains five valence electrons available to form strong valence bonds with carbon atoms, NG exhibits different properties as compared with that of the pristine GR. There are three commonly bonding configurations of nitrogen atom that could be found in NG, that is pyridinic N, porrolic $\mathrm{N}$ and graphitic N. ${ }^{[12]}$ Wang et al. reviewed the recent progresses of NG and its potential applications. ${ }^{[13]}$ Shao et al. applied NG in the electrochemical applications such as electrochemical energy devices and biosensors. ${ }^{[14]}$ Wang et al. applied NG in electrochemical biosensing for glucose. ${ }^{[15]}$ Sun et al. fabricated a NG modified carbon ionic liquid electrode for the detection of rutin. ${ }^{[16]}$ Therefore NG has the potential applications in the fields of electrochemistry and electrochemical sensor.

Dopamine (DA) is a kind of biogenic amine that acts as neurotransmitter in the physiological system. It has been 
widely studied due to its important functions in renal, cardiovascular, hormonal and nervous systems. ${ }^{[17]}$ Because of the electroactivity of DA in the biological samples, electrochemical detection of DA has gained increasing attentions. ${ }^{[18]}$ Kan et al. applied a multi-walled carbon nanotube composite with homogeneous molecularly imprinted polymers outer layer modified glassy carbon electrode (GCE) for the recognization of DA in presence of ascorbic acid (AA). ${ }^{[19]}$ Oztekin et al. used a copper nanoparticles (CuNP) modified GCE for the selective determination of DA in the presence of $A A$, uric acid and $p$-acetamidophenol. ${ }^{[20]}$ Niu et al. applied a 3,4,9,10-perylene tetracarboxylic acid functionalized GR sheets/multi-walled carbon nanotubes/ionic liquid modified electrode for the DA detection. ${ }^{[21]}$ These electrochemical sensors with different kinds of modifiers have exhibited the advantages including cheap instruments, fast response, low cost, high sensitivity and good selectivity.

In this paper NG nanosheet was used to modify the commonly used GCE and the fabricated NG/GCE was further applied to the sensitive electrochemical detection of DA. Due to the electrocatalytic activity of NG with its unique structure and large surface area, DA exhibited an enhanced electrochemical response on NG/GCE with a pair of welldefined redox peaks appeared. Electrochemical behaviors of DA were carefully investigated on NG/GCE with the electrochemical parameters calculated. The proposed method was further applied to the DA injection samples detection with satisfactory results.

\section{EXPERIMENTAL}

\section{Apparatus and Reagents}

Electrochemical measurements were performed on a $\mathrm{CHI}$ 750B electrochemical workstation (Shanghai CH Instrument, China) with conventional three-electrode cell. A bare GCE or NG/GCE was used as the working electrode. A saturated calomel electrode (SCE) and a platinum wire were used as the reference and counter electrodes, respectively. Scanning electron microscopy (SEM) was recorded on a JSM-6700F scanning electron microscope (Japan Electron Company, Japan). Transmission electron microscopy (TEM) image was acquired by a JEM-2100 transmission electron microscope (JEOL, Japan) at a $200 \mathrm{kV}$ acceleration potential.

Dopamine (DA) hydrochloride was purchased from Aladdin Chemical Reagent Co. Ltd. (Shanghai, China). NG was synthesized in accordance with recent reported procedure. ${ }^{[22]}$ A $1.0 \mathrm{mg} \mathrm{mL}^{-1} \mathrm{NG}$ dispersion solution was prepared by ultrasonication for 2 hours in water. $0.1 \mathrm{~mol} \mathrm{~L}^{-1}$ phosphate buffer solution (PBS) was used as the supporting electrolyte. All other reagents were of analytical grade and all aqueous solutions were prepared with doubly distilled water.

\section{Preparation of NG/GCE}

Prior to use, GCE with a diameter of $3 \mathrm{~mm}$ was polished on a polishing cloth with 1.0, 0.3 and $0.05 \mu \mathrm{m}$ alumina powder, respectively, and rinsed with doubly distilled water, followed by sonication in ethanol solution and doubly distilled water successively. Then the electrode was dried in a stream of nitrogen. Afterwards, a $5 \mu \mathrm{L}$ of $0.5 \mathrm{mg} \mathrm{mL}^{-1} \mathrm{NG}$ solution was dropped to fully cover the surface of the polished GCE and dried at room temperature to get the modified electrode (NG/GCE).

\section{Electrochemical Detection}

A certain concentration of DA solution prepared with 0.1 mol L-1 PBS of $\mathrm{pH}=7.0$ was added into a $10 \mathrm{~mL}$ electrochemical cell and the three-electrode system was inserted into the solution. Then cyclic voltammetry (CV) was performed in the potential range from $-0.2 \mathrm{~V}$ to $0.6 \mathrm{~V}$ at the scan rate of $100 \mathrm{mV} \mathrm{s}^{-1}$. Differential pulse voltammetric (DPV) measurements were carried out for the quantitative analysis with the parameters set as: step increment potential of $0.004 \mathrm{~V}$, pulse amplitude of $0.05 \mathrm{~V}$, pulse width of $0.05 \mathrm{~s}$, and pulse period of $0.2 \mathrm{~s}$.

\section{RESULTS AND DISCUSSION}

\section{Characteristics of NG/GCE}

Figure $1 A$ is showing the SEM image of NG/GCE with inset as the TEM image of NG. As shown in the inset of Figure $1 \mathrm{~A}$, NG was present as the nanosheet with some folded and rippled. After NG was modified on the surface of GCE, large amounts of nanosheet was present with porous structure appeared, indicating that the presence of NG nanosheet on the electrode surface resulted in the increase of the effective electrode area.

Electrochemical behaviors of different modified electrodes were further investigated in the ferricyanide solution with the cyclic voltammograms shown in Figure 1B. The redox peak currents of $\left[\mathrm{Fe}(\mathrm{CN})_{6}\right]^{3-/ 4-}$ on GCE (curve a) was much smaller than that of NG/GCE (curve b) with the decrease of the peak-to-peak separation $\left(\Delta E_{\mathrm{p}}\right)$ on NG/GCE. The results indicated that the presence of NG on the GCE surface can greatly enhance the electrochemical responses, which may be attributed to the specific characteristics of NG that can accelerate the electron transfer rate. EIS experiments were further carried out with the results shown in Figure 1C. The semicircular portion at high frequencies in the Nyquist diagrams corresponds to the electron-transferlimited process and its diameter is equal to the electrontransfer resistance $\left(R_{\mathrm{et}}\right)$, which controls the electrontransfer kinetics of the redox probe at the electrode. Meanwhile, the linear part at lower frequencies corresponds to the diffusion process. The Randles circuit model is 
chosen to fit the impedance data got in the experiment. The $R_{\text {et }}$ value of GCE was got as $76.1 \Omega$ (curve a), which was bigger than that of NG/GCE (46.6 $\Omega$ ). The results indicated that the decrease of the interfacial resistance was due to the presence of NG. Electrochemical behaviors of ferricyanide on NG/GCE were further investigated with the changes of scan rate and the corresponding cyclic voltammograms were listed in Figure 1D. It can be seen that a pair of well-defined redox peaks appeared at different scan rate. The redox peak currents exhibited good linear relationships with scan rate, and the linear regression equations were got as $I_{\mathrm{pc}} / \mu \mathrm{A}=66.99 u^{1 / 2} /\left(\mathrm{V} \mathrm{s}^{-1}\right)-3.13(n=11, \gamma=$ 0.999) and $I_{\mathrm{pa}} / \mu \mathrm{A}=-65.76 u^{1 / 2} /\left(\mathrm{V} \mathrm{s}^{-1}\right)+2.74(n=11, \gamma=$ $0.998)$. So the electrochemical reaction of ferricyanide on NG/GCE was a diffusional controlled process, which could be attributed to the presence of high conductive NG with large surface area on the electrode surface. NG has been proven to exhibit excellent electrocatalytic activity with fast electron transfer rate. The relationships of redox peak potentials with $\ln u$ were also obtained with the following regression equations as $E_{\mathrm{pc}} / \mathrm{V}=-0.037 \ln \left(u /\left(\mathrm{V} \mathrm{s}^{-1}\right)\right)+0.21$ $(n=11, \gamma=0.998)$ and $E_{\mathrm{pa}} / \mathrm{V}=0.064 \ln \left(u /\left(\mathrm{V} \mathrm{s}^{-1}\right)\right)+0.31(n$ $=11, \gamma=0.997)$. Based on Nicholson's equations: ${ }^{[23]}$
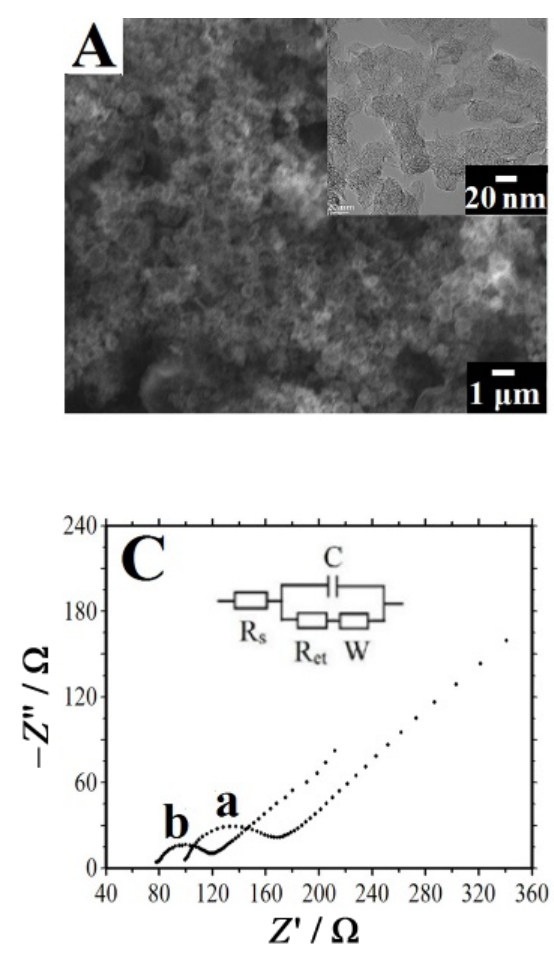

$$
\begin{aligned}
& E_{p a}=E^{0^{\prime}}+m\left[0.78+\ln \left(D^{1 / 2} k_{s}^{-1}\right)-0.5 \ln m\right]+\frac{m}{2} \ln u, m=\frac{R T}{(1-\alpha) n F} \\
& E_{p c}=E^{0^{\prime}}-m^{\prime}\left[0.78+\ln \left(D^{1 / 2} k_{s}^{-1}\right)-0.5 \ln m^{\prime}\right]-\frac{m^{\prime}}{2} \ln u, m^{\prime}=\frac{R T}{\alpha n F} \\
& \log k_{s}=\alpha \log (1-\alpha)+(1-\alpha) \log \alpha-\log \frac{R T}{n F u}-\frac{(1-\alpha) \alpha F \Delta E_{p}}{2.3 R T}
\end{aligned}
$$

The electrochemical parameters, such as the electron transfer coefficients $(\alpha)$, the electron transfer number $(n)$ and the apparent heterogeneous electron transfer rate constant $\left(k_{s}\right)$, of the electrochemical reaction of ferricyanide were calculated as $0.34,0.98$, and $1.04 \mathrm{~s}^{-1}$, respectively.

\section{Cyclic Voltammograms of DA on NG/GCE}

Cyclic voltammograms of $1.0 \times 10^{-4} \mathrm{~mol} \mathrm{~L}^{-1} \mathrm{DA}$ on different working electrodes in PBS of $\mathrm{pH}=7.0$ were recorded with the results shown in Figure 2. It can be seen that a pair of well-defined redox peaks appeared on the voltammograms, which was the typical results of DA electrochemical reaction. ${ }^{[24]}$ On GCE the redox peak potentials were located at $0.246 \vee\left(E_{\mathrm{pa}}\right)$ and $0.139 \mathrm{~V}\left(E_{\mathrm{pc}}\right)$ with the redox peak
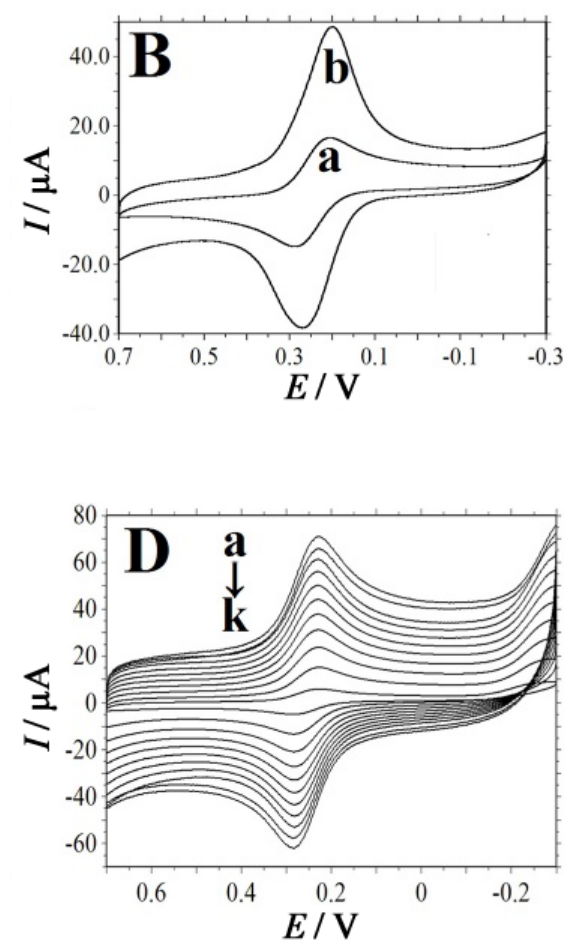

Figure 1. (A) SEM image of NG/GCE (inset is the TEM of NG); (B) Cyclic voltammograms of (a) GCE and (b) NG/GCE in the presence of a $1.0 \mathrm{mmol} \mathrm{L}^{-1} \mathrm{~K}_{3}\left[\mathrm{Fe}(\mathrm{CN})_{6}\right]$ and $0.5 \mathrm{~mol} \mathrm{~L}^{-1} \mathrm{KCl}$ solution with the scan rate of $100 \mathrm{mV} \mathrm{s}^{-1}$; (C) EIS of (a) GCE and (b)

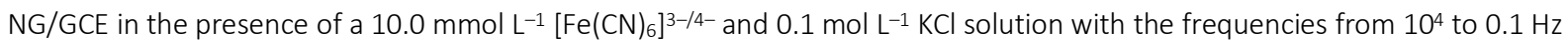
(Inset is the Randles circuit model in the cell); (D) Cyclic voltammograms of NG/GCE in a $1.0 \mathrm{mmol} \mathrm{L}^{-1} \mathrm{~K}_{3}\left[\mathrm{Fe}(\mathrm{CN})_{6}\right]$ and $0.5 \mathrm{~mol}$ $\mathrm{L}^{-1} \mathrm{KCl}$ solution with different scan rates (form a to $\mathrm{k}: 0.50,0.45,0.40,0.35,0.30,0.25,0.20,0.15,0.10,0.05,0.01 \mathrm{~V} \mathrm{~s}^{-1}$, respectively). 


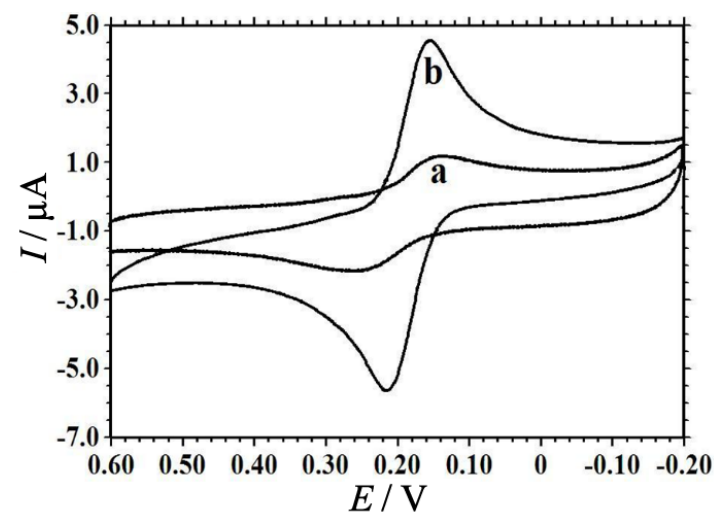

Figure 2. Cyclic voltammograms of $1.0 \times 10^{-4} \mathrm{~mol} \mathrm{~L}^{-1} \mathrm{DA}$ on (a) GCE and (b) NG/GCE at a scan rate of $100 \mathrm{mV} \mathrm{s}^{-1}$ in PBS of $\mathrm{pH}=7.0$.

current as $1.308 \mu \mathrm{A}\left(I_{\mathrm{pa}}\right)$ and $0.992 \mu \mathrm{A}\left(I_{\mathrm{pc}}\right)$. The peak-topeak separation $(\Delta E)$ was calculated as $107 \mathrm{mV}$ with the $I_{\mathrm{pa}} / I_{\mathrm{pc}}$ value as 1.318 , which was a typical result of quasireversible reaction. Whereas on NG/GCE the redox peak potentials were located at $0.218 \mathrm{~V}\left(E_{\mathrm{pa}}\right)$ and $0.156 \mathrm{~V}\left(E_{\mathrm{pc}}\right)$ with the redox peak currents as $5.231 \mu \mathrm{A}\left(I_{\mathrm{pa}}\right)$ and $4.897 \mu \mathrm{A}$ $\left(I_{\mathrm{pc}}\right)$. The values of $\Delta E_{\mathrm{p}}$ and $I_{\mathrm{pa}} / I_{\mathrm{pc}}$ were got as $62 \mathrm{mV}$ and 1.068 , indicating a more reversible electrochemical process. Also the redox peak current increased for 4.00 and 5.03 times than that of GCE, respectively, which was the typical electrocatalytic effect. The result was attributed to the presence of NG on the surface of GCE. Chemical doping is a strategy for the preparation of functionalized carbon materials and the presence of nitrogen in the carbon structure can modulate the properties. ${ }^{[25]}$ Nitrogen atom has comparable atom size with carbon, and it contains five valence electrons bonds with carbon atom. So the chemical doping of nitrogen can partly restore the conductivity of $\mathrm{GR}$. The presence of pyridinic $\mathrm{N}$, pyrrolic $\mathrm{N}$ and quaternary $\mathrm{N}$ on the GR surface exhibited certain catalytic ability. ${ }^{[26]}$ And the two-dimensional structure of GR still remained with large surface area. Also NG has aromatic rings with rich delocalized $\pi$ electrons, which can interact with the DA molecules that have a specific aromatic ring through $\pi-\pi$ stacking force. So the presence of NG on the electrode surface exhibited excellent electrocatalytic activity to the DA electrooxidation with the decrease of the overpotentials and the increase of the redox peak currents. Therefore NG/GCE is a suitable working electrode for the sensitive detection of DA.

\section{Influence of Scan Rate}

The kinetics of the electrode reaction were investigated by studying the effect of scan rate on the electrochemical responses of DA on NG/GCE. Figure $3 A$ showed the cyclic voltammograms of $1.0 \times 10^{-4} \mathrm{~mol} \mathrm{~L}^{-1} \mathrm{DA}$ on NG/GCE in the scan rate range from 20.0 to $500.0 \mathrm{mV} \mathrm{s}^{-1}$. It can be observed that a pair of well-defined redox peaks appeared at different scan rate with the redox peak potential and currents changed gradually. With the increase of scan rate the redox peak currents increased and good linear relationships of the redox peak current $\left(I_{\mathrm{p}}\right)$ with the square root of scan rate $\left(u^{1 / 2}\right)$ were obtained. The linear regression equations were got as $I_{\mathrm{pa}} / \mu \mathrm{A}=-6.31 u^{1 / 2} /\left(\mathrm{V} \mathrm{s}^{-1}\right)-1.28(n=15, \gamma=0.997)$ and $I_{\mathrm{pc}} / \mu \mathrm{A}=9.38 u^{1 / 2} /\left(\mathrm{V} \mathrm{s}^{-1}\right)+0.57(n=15, \gamma=0.998)$, illustrating a diffusional controlled process. The result indicated that electrode reaction of DA was fast and DA molecules diffused to the electrode surface could take place electrochemical reaction quickly, which could be attributed to the high conductivity of NG on the electrode surface. The relationship of redox peak potentials and $\ln v$ was also constructed and the linear regression equations were got as $E_{\mathrm{pa}} / \mathrm{V}=0.0274 \ln \left(u /\left(\mathrm{V} \mathrm{s}^{-1}\right)\right)+0.3205(n=15, \gamma$ $=0.997)$ and $E_{\mathrm{pc}} / \mathrm{V}=-0.0205 \ln \left(u /\left(\mathrm{V} \mathrm{s}^{-1}\right)\right)+0.0910(n=10$, $\gamma=0.998)$. According to the Nicholson's equations, ${ }^{[23]}$ the electrochemical parameters of DA on NG/GCE, such as the charge transfer coefficient $(\alpha)$, the number of electron transfer $(n)$ and the electrode reaction rate constant $\left(k_{s}\right)$, were calculated as $0.49,2.2$ and $1.174 \mathrm{~s}^{-1}$.
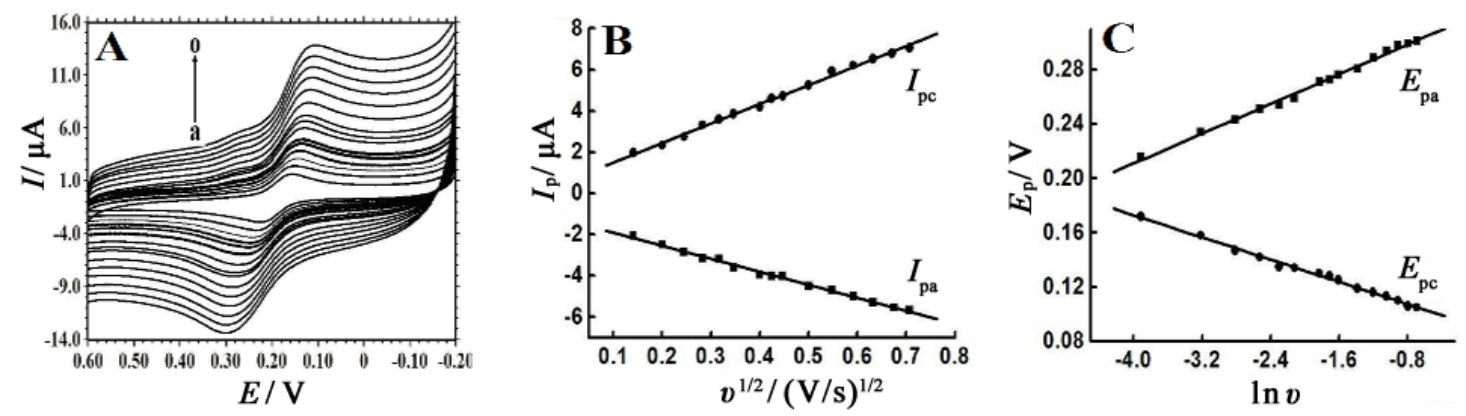

Figure 3. (A) Cyclic voltammograms of $1.0 \times 10^{-4} \mathrm{~mol} \mathrm{~L}^{-1} \mathrm{DA}$ on NG/GCE at different scan rate (from a to $0: 0.04,0.06,0.08,0.10$, $\left.0.12,0.14,0.16,0.18,0.20,0.25,0.30,0.35,0.40,0.45,0.50 \mathrm{~V} \mathrm{~s}^{-1}\right)$; (B) Linear relationship of the redox peak currents $\left(I_{\mathrm{p}}\right)$ vs. $U^{1 / 2}$; (C) Linear relationship between the redox peak potentials $\left(E_{\mathrm{p}}\right)$ and $\ln U$. 


\section{Influence of Buffer pH}

The influence of buffer $\mathrm{pH}$ on the cyclic voltammetric responses of $1.0 \times 10^{-4} \mathrm{~mol} \mathrm{~L}^{-1} \mathrm{DA}$ was investigated in the $\mathrm{pH}$ range from 4.5 to 9.0 , The relationships of the oxidation peak current and the formal peak potential $\left(E^{0^{\prime}}\right)$ with buffer $\mathrm{pH}$ were plotted with the results shown in Figure 4. The maximum value of the oxidation peak current appeared at buffer of $\mathrm{pH}=7.0$ and decreased gradually with the further increase of buffer $\mathrm{pH}$ (Figure 4A). Therefore, $\mathrm{pH}=7.0$ was selected as the optimal $\mathrm{pH}$ for detection in the following experiments. With the increase of the buffer $\mathrm{pH}$, the value of $E^{0^{\prime}}$ also shifted to the negative direction, indicating that protons participated in the reaction. The relationship between $E^{0^{\prime}}$ and $\mathrm{pH}$ was calculated as $E^{0^{\prime}} / \mathrm{V}=-0.052 \mathrm{pH}+0.54$ ( $n=10, v=0.996$ ) (Figure 4B). The slope value of $-52.0 \mathrm{mV}$ $\mathrm{pH}^{-1}$ was close to the theoretical value of $-59.0 \mathrm{mV} \mathrm{pH}^{-1}$, [27] indicated that the ratio of electrons and protons taking part in the electrode reaction was $1: 1$. According to the above result of the number of electron transferred was calculated as 2 , so the protons involved in the electrode reaction was also got as 2 .
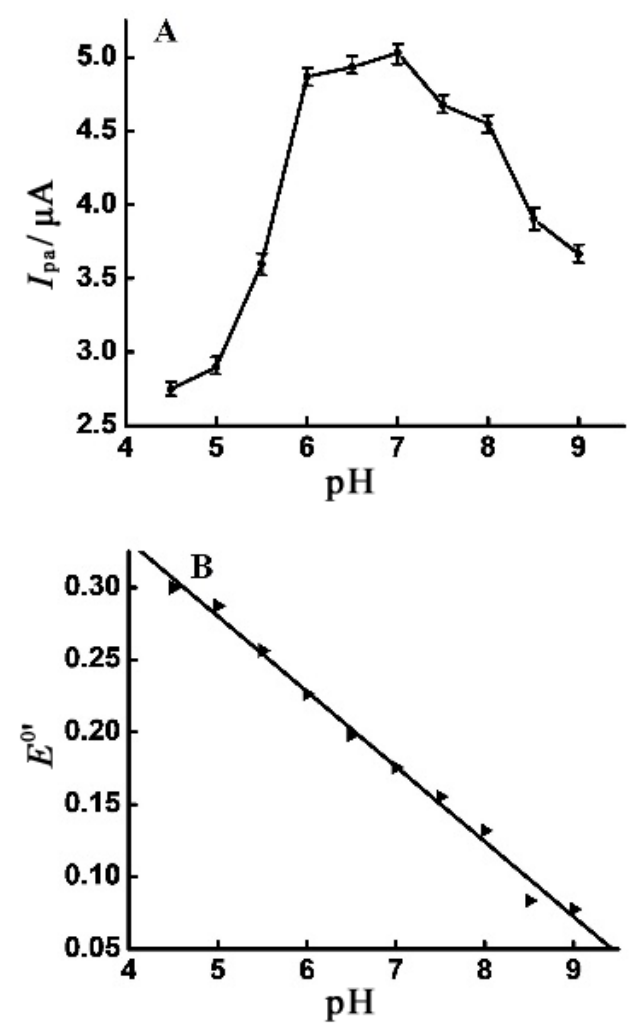

Figure 4. (A)The relationship between the oxidation peak current $\left(I_{\mathrm{pa}}\right)$ and $\mathrm{pH}$; (B) Linear relationship of the formal peak potential $\left(E^{\mathrm{O}^{\prime}}\right)$ and $\mathrm{pH}$.

\section{Chronocoulometric Experiments}

Since the electrode reaction was diffusion-controlled, the chronocoulometric response of DA on NG/GCE was investigated to calculate the diffusional coefficient $(D)$. Figure $5 A$ showed the chronocoulometric curves of NG/GCE in the given solutions, and a good linear relationship between $Q$ and $t^{1 / 2}$ was shown in Figure $5 \mathrm{~B}$. According to Anson's equation: ${ }^{[28]} Q=2 n F A D^{1 / 2} c t^{1 / 2} / \pi^{1 / 2}+Q_{\mathrm{dl}}+n F \Gamma$, the $D$ value of DA was calculated as $9.86 \times 10^{-5} \mathrm{~cm}^{2} \mathrm{~s}^{-1}$.

\section{Calibration Curve}

Under the selected conditions differential pulse voltammetry (DPV) was used to quantitative determination of DA. The DPV oxidation peak currents increased linearly with DA concentrations in the range from $8.0 \times 10^{-7} \mathrm{~mol} \mathrm{~L}^{-1}$ to $8.0 \times 10^{-4} \mathrm{~mol} \mathrm{~L}^{-1}$ with the typical differential pulse voltammograms shown in Figure 6A. The relationship between the oxidation peak current $\left(I_{\mathrm{pa}}\right)$ and DA concentration $(C)$ was calculated as $I_{\mathrm{pa}} / \mu \mathrm{A}=0.167 \mathrm{C} / \mu \mathrm{mol} \mathrm{\textrm {L } ^ { - 1 }}$ - 0.239 ( $n=13, y=0.9997$ ) (Figure $6 \mathrm{~B}$ ) with the detection limit as $2.55 \times 10^{-7} \mathrm{~mol} \mathrm{~L}^{-1}(3 \sigma)$. The detection
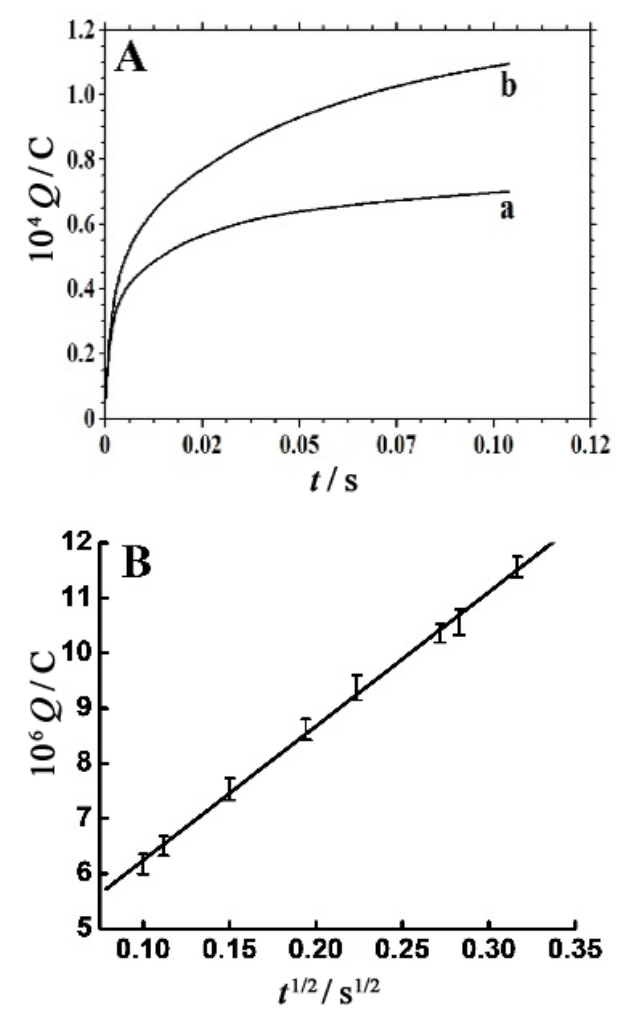

Figure 5. (A) Chronocoulometric curves of NG/GCE in (a) pH $=7.0$ buffer solution and (b) $\mathrm{pH}=7.0$ buffer solution containing $1.0 \times 10^{-4} \mathrm{~mol} \mathrm{~L}^{-1} \mathrm{DA}$; (B) Linear relationship between $Q$ and $t^{1 / 2}$. 

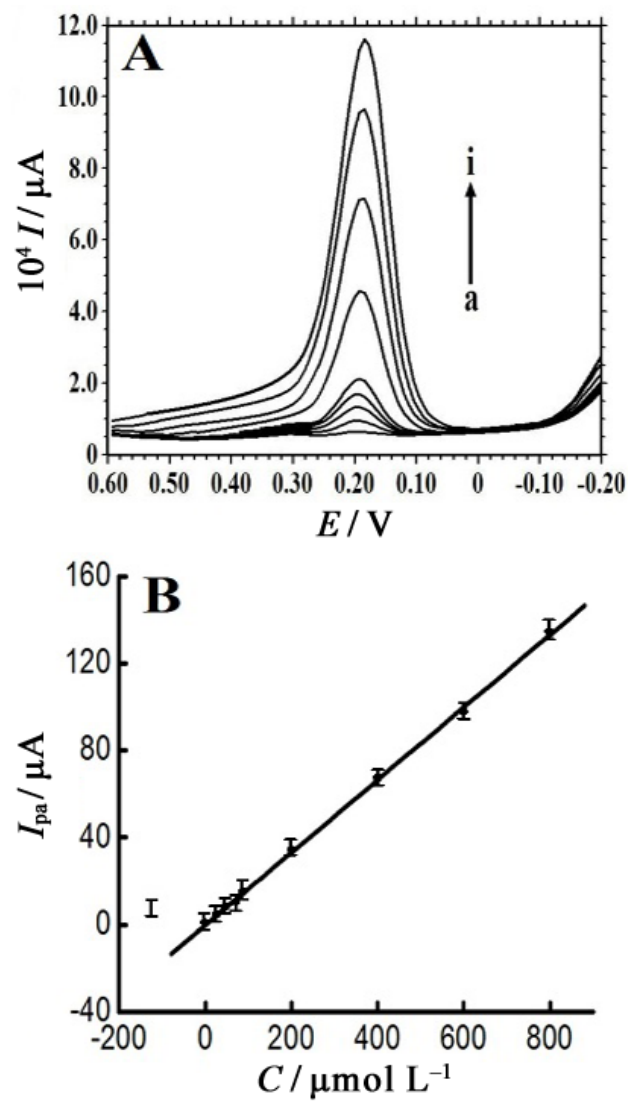

Figure 6. (A)Typical differential pulse voltammograms of various concentrations DA on NG/GCE (from a to i: 4, 6, 8, 20, 40, 60, 80, 200, $400 \mu \mathrm{mol} \mathrm{L}^{-1}$, respectively); (B) The linear relationship of the oxidation peak current with the DA concentration. limit was smaller than some previous reported works such as $\mathrm{Ni} / \mathrm{Al}$ layered double hydroxide modified carbon ionic liquid electrode $\left(5.0 \times 10^{-6} \mathrm{~mol} \mathrm{~L}^{-1}\right)$, ${ }^{[29]} \mathrm{PtAu}$ hybrid film modified GCE $\left(5.0 \times 10^{-6} \mathrm{~mol} \mathrm{~L}^{-1}\right),{ }^{[30]}$ and GR modified GCE $\left(2.64 \times 10^{-6} \mathrm{~mol} \mathrm{~L}^{-1}\right),{ }^{[31]} \mathrm{GR}$ and Au nanoparticles modified GCE $\left(1.86 \times 10^{-6} \mathrm{~mol} \mathrm{~L}^{-1}\right),{ }^{[32]} \mathrm{TiO}_{2}$-GR modified GCE $\left(2.0 \times 10^{-6}\right.$ $\left.\mathrm{mol} \mathrm{L}{ }^{-1}\right),{ }^{[33]}$ and graphene oxide modified GCE $\left(0.27 \times 10^{-6}\right.$ mol L $\left.{ }^{-1}\right){ }^{[34]}$ So the presence of NG on the GCE surface exhibited excellent electrocatalytic activity to the oxidation of DA with increased sensitivity.

\section{Interferences}

The influences of some interfering materials on the determination of $1.0 \times 10^{-4} \mathrm{~mol} \mathrm{~L}^{-1} \mathrm{DA}$ were investigated. The proposed method showed good selectivity for DA detection without the interferences from common coexisting materials in samples, such as ions (e.g. $\mathrm{Zn}^{2+}, \mathrm{K}^{+}, \mathrm{Ca}^{2+}, \mathrm{Cl}^{-}, \mathrm{Na}^{+}$, $\left.\mathrm{Cu}^{2+}\right)$, glucose, deoxyribonucleic acid, ribonucleic acid etc. with signal changes below $\pm 5 \%$ (as shown in the Table 1 ). Thus, electrochemical signals of these substances did not disturb that of DA and hence NG/GCE showed good selectively with the ability to distinguish other electrochemical responses.

\section{Sample Determination}

The developed method was applied to the detection of the content in DA injection samples, which were diluted and detected by the proposed procedure with the results shown in Table 2. Also the standard addition method was used to calculate the recovery. From Table 2 it can be seen that the DA injection samples were detected with satisfactory results. The relative standard deviation (RSD) values

Table 1. Influence of interfering materials on the determination of $1.0 \times 10^{-4} \mathrm{~mol} \mathrm{~L}^{-1} \mathrm{DA}(n=6)$

\begin{tabular}{cccccc}
\hline Coexisting substance & $\mathrm{C} / \mathrm{mg} \mathrm{L}^{-1}$ & Relative error /\% & Coexisting substance & $\mathrm{C} / \mathrm{mol} \mathrm{L}^{-1}$ & Relative error /\% \\
\hline Glucose & 10.0 & 1.98 & Pyrocatechol & $1.0 \times 10^{-5}$ & -1.65 \\
Deoxyribonucleic acid & 10.0 & -3.47 & $\mathrm{Zn}^{2+}$ & $1.0 \times 10^{-5}$ & -2.48 \\
Ribonucleic acid & 10.0 & 1.45 & $\mathrm{~K}^{+}$ & $1.0 \times 10^{-2}$ & -2.67 \\
L-arginine & 10.0 & 2.53 & $\mathrm{Ca}^{2+}$ & $1.0 \times 10^{-5}$ & -3.59 \\
L-cysteine & 10.0 & 0.95 & $\mathrm{Cu}^{2+}$ & $1.0 \times 10^{-4}$ & 1.56 \\
Sodium dodecyl & 10.0 & 0.46 & $\mathrm{Na}^{+}$ & $1.0 \times 10^{-4}$ & 3.85 \\
sulfate & 10.0 & -1.68 & $\mathrm{Cl}^{-}$ & $1.0 \times 10^{-2}$ & -2.69 \\
Glycine & & & & & \\
\hline
\end{tabular}

Table 2. Determination of DA in the injection sample $(n=6)$

\begin{tabular}{ccccccc}
\hline Sample & $C$ (Specified) $/ \mathrm{mol} \mathrm{L}^{-1}$ & $C$ (Detected) $/ \mathrm{mol} \mathrm{L}^{-1}$ & $C$ (Added) $/ \mathrm{mol} \mathrm{L}^{-1}$ & $C$ (Detected) $/ \mathrm{mol} \mathrm{L}^{-1}$ & RSD $/ \%$ & Recovery \% \\
\hline 1 & $10.0 \times 10^{-5}$ & $11.05 \times 10^{-5}$ & $10.0 \times 10^{-5}$ & $10.47 \times 10^{-5}$ & 1.76 & 104.7 \\
2 & $30.0 \times 10^{-5}$ & $31.2 \times 10^{-5}$ & $10.0 \times 10^{-5}$ & $10.42 \times 10^{-5}$ & 2.79 & 104.2 \\
3 & $50.0 \times 10^{-5}$ & $49.1 \times 10^{-5}$ & $10.0 \times 10^{-5}$ & $9.75 \times 10^{-5}$ & 1.34 & 97.5 \\
\hline
\end{tabular}


were in the range from $1.76 \%$ to $2.79 \%$ with the recovery in the range from $97.5 \%$ to $104.7 \%$, which indicated that this modified electrode could be used for the DA samples detection.

\section{Stability and Repeatability of the Modified Electrode}

The stability and repeatability of the modified electrode were evaluated. The RSD of eleven successive scans for $1.0 \times 10^{-4} \mathrm{~mol} \mathrm{~L}^{-1} \mathrm{DA}$ was got as $1.89 \%$, indicating good reproducibility of NG/GCE. In dry state, only $2.4 \%$ loss of DPV peak current value was found even after two weeks storage, indicating the good stability of NG/GCE. The repeatability of eight independently fabricated electrodes showed a satisfactory RSD value of $3.72 \%$ for the detection of $1.0 \times 10^{-4} \mathrm{~mol} \mathrm{~L}^{-1} \mathrm{DA}$. All these results indicated that NG/GCE was stable for the electrochemical application.

\section{CONCLUSION}

In the paper a NG modified GCE was fabricated and further used for investigation on the electrochemistry of DA in detail. Electrochemical behaviors of DA on NG/GCE were carefully studied with the electrochemical parameters calculated. The presence of NG on the electrode showed good promotion to the electro-oxidation of DA, which could be attributed to the specific properties and unique structure of NG. Based on the oxidation peak current, DA can be detected in the concentration range from $8.0 \times 10^{-7} \mathrm{~mol} \mathrm{~L}^{-1}$ to $8.0 \times 10^{-4} \mathrm{~mol} \mathrm{~L}^{-1}$ with the detection limit of $2.55 \times 10^{-7}$ $\mathrm{mol} \mathrm{L}^{-1}(3 \sigma)$ by differential pulse voltammetry. Under the selected conditions a new electrochemical method for DA detection was further established and successfully applied to DA injection sample detection with satisfactory results.

Acknowledgment. This work was supported by the National Natural Science Foundation of China (51172113, 21365010), the Nature Science Foundation of Hainan Province (20162031), the Science and Research Key Project of Universities of Hainan Province (Hnky2016ZD-10), the International Science and Technology Cooperation Project of Hainan Province (KJHZ2015-13).

\section{REFERENCES}

[1] A. K. Geim, K. S. Novoselov, Nat. Mater. 2007, 6, 183.

[2] E. V. Iski, E. N. Yitamben, L. Gao, N. P. Guisinger, Adv. Funct. Mater. 2013, 23, 2554.

[3] V. Singh, D. Joung, L. Zhai, S. Das, S. I. Khondaker, S. Seal, Prog. Mater. Sci. 2011, 56, 1178.

[4] N. O. Weiss, H. L. Zhou, L. Liao, Y. Liu, S. Jiang, Y. Huang, X. F. Duan, Adv. Mater. 2012, 24, 5782.

[5] T. Gan, S. Hu, Microchim. Acta 2011, 175, 1.
[6] K. R. Ratinac, W. Yang, J. J. Gooding, P. Thordarson, F. Braet, Electroanal. 2011, 23, 803.

[7] D. Chen, L. H. Tang, J. H. Li, Chem. Soc. Rev. 2010, 39, 3157.

[8] D. A. C. Brownson, D. K. Kampouris, C. E. Banks, Chem. Soc. Rev. 2012, 41, 6944.

[9] R. T. Lv, Q. Li, A. R. Botello-Mendez, T. Hayashi, B. Wang, A. Berkdem ir, Q. Z. Hao, A. L. Elias, R. CruzSilva, H. R. Gutierrez, Y. A. Kim, H. Muramatsu, J. Zhu, M. Endo, H. Terrones, J. C. Charlier, M. H. Pan, M. Terrones, Sci. Rep-UK 2012, 2, 586.

[10] Z. H. Sheng, L. Shao, J. J. Chen, W. J. Bao, F. B. Wang, X. H. Xia, ACS Nano 2011, 5, 4350.

[11] L. S. Panchakarla, K. S. Subrahmanyam, S. K. Saha, A. Govindaraj, H. R. Krishnamurthy, U. V. Waghmare, C. N. R. Rao, Adv. Materi. 2009, 21, 4726.

[12] L. Sun, L. Wang, C. G. Tian, T. X. Tan, Y. Xie, K. Y. Shi, M. T. Li, H. G. Fu, RSC Adv. 2012, 2, 4498.

[13] H. B. Wang, T. Maiyalagan, X. Wang, ACS Catal. 2012, 2, 781.

[14] Y. Y. Shao, S. Zhang, M. H. Engelhard, G. S. Li, G. C. Shao, Y. Wang, J. Liu, I. A. Aksay, Y. H. Lin, J Mater. Chem. 2010, 20, 7491.

[15] Y. Wang, Y. Y. Shao, D. W. Matson, J. H. Li, Y. H. Lin, ACS Nano 2010, 4, 1790.

[16] W. Sun, L. F. Dong, Y. X. Lu, Y. Deng, J. H. Yu, X. H. Sun, Q. Q. Zhu, Sensor. Actuat. B-Chem 2014, 199, 36.

[17] R. P. Deo, N. S. Lawrence, J. Wang, Analyst 2004, $129,1076$.

[18] K. Jackowska, P. Krysinski, Anal. Bioanal. Chem. 2013, 405, 3753.

[19] X. W. Kan, Y. Zhao, Z. R. Geng, Z. L. Wang, J. J. Zhu, J. Phys. Chem. C 2008, 112, 4849.

[20] Y. Oztekin, M. Tok, E. Bilici, L. Mikoliunaite, Z. Yazicigil, A. Ramanaviciene, A. Ramanavicius, Electrochim. Acta 2012, 76, 201.

[21] X. L. Niu, W. Yang, H. Guo, J. Ren, J. Z. Gao, Biosens. Bioelectron. 2013, 41, 225.

[22] H. Z. Dong, J. C Bai, Q. Q. Zhu, J. H. Yu, L. Y. Yu, L. F. Dong, J. Renew. Sustain. Ener. 2013, 5, 021405.

[23] R. S. Nicholson, I. Shain, Anal. Chem. 1964, 36, 706.

[24] W. Sun, X. Z. Wang, Y. H. Wang, X. M. Ju, L. Xu, G. J. Li, Z. F. Sun, Electrochim. Acta 2013, 87, 317.

[25] Y. C. Ma, A. S. Foster, A. V. Krasheninnikov, R. M. Nieminen, Phys. Rev. B 2005, 72, 205416.

[26] P. H. Matter, L. Zhang, U. S. Ozkan, J. Catal. 2006, 239, 83.

[27] R. S. Nicholson, Anal. Chem. 1965, 37, 1351.

[28] F. C. Anson, Anal. Chem. 1964, 36, 932.

[29] Z. H. Zhu, L. N. Qu, Y. Q. Guo, Y. Zeng, W. Sun, X. T. Huang, Sensor. Actuat. B-Chem. 2010, 151, 146.

[30] S. Thiagarajan, and S. M. Chen, Talanta 2007, 74, 212. 
[31] Y. R. Kim, S. Bong, Y. J. Kang, Y. Yang, R. K. Mahajan, J. S. Kim, H. Kim, Biosens. Bioelectron. 2010, 25, 2366.

[32] J. Li, J. Yang, Z. J. Yang, Y. F. Li, S. H. Yu, Q. Xu, X. Y. Hu, Anal. Methods 2012, 4, 1725.
[33] Y. Fan, H. T. Lu, J. H. Liu, C. P. Yang, Q. S. Jing, Y. X. Zhang, X. K. Yang, K. J. Huang, Colloids Surf. B 2011, $83,78$.

[34] F. Gao, X. L. Cai, X. Wang, C. Gao, S. L. Liu, F. Gao, Q. X. Wang, Sensor. Actuat. B-Chem. 2013, 186, 380. 